\title{
Drivers from the deep: the contribution of collicular input to thalamocortical processing
}

\author{
Robert H. Wurtz*, Marc A. Sommer and James Cavanaugh \\ Laboratory of Sensorimotor Research, National Eye Institute, National Institutes of Health, \\ Bethesda, MD 20892-4435, USA
}

\begin{abstract}
A traditional view of the thalamus is that it is a relay station which receives sensory input and conveys this information to cortex. This sensory input determines most of the properties of first order thalamic neurons, and so is said to drive, rather than modulate, these neurons. This holds as a rule for first order thalamic nuclei, but in contrast, higher order thalamic nuclei receive much of their driver input back from cerebral cortex. In addition, higher order thalamic neurons receive inputs from subcortical movement-related centers. In the terminology popularized from studies of the sensory system, can we consider these ascending motor inputs to thalamus from subcortical structures to be modulators, subtly influencing the activity of their target neurons, or drivers, dictating the activity of their target neurons? This chapter summarizes relevant evidence from neuronal recording, inactivation, and stimulation of pathways projecting from the superior colliculus through thalamus to cerebral cortex. The study concludes that many inputs to the higher order nuclei of the thalamus from subcortical oculomotor areas - from the superior colliculus and probably other midbrain and pontine regions - should be regarded as motor drivers analogous to the sensory drivers at the first order thalamic nuclei. These motor drivers at the thalamus are viewed as being at the top of a series of feedback loops that provide information on impending actions, just as sensory drivers provide information about the external environment.
\end{abstract}

\section{Introduction}

The thalamus has long been recognized as a gateway to the cerebral cortex for sensory information flowing into the brain from the periphery (Sherman and Guillery, 2001; Jones, 2002). Everything we know about the world necessarily results from the inflow of such sensory information. The same may be true about movement information; it seems likely that much, perhaps all, of the precise information our brain receives regarding our actions comes from

\footnotetext{
*Corresponding author. Tel.: +1-301-496-7170; Fax: +1-301402-0511; E-mail: bob@1sr.nei.nih.gov
}

feedback to the cerebral cortex through the thalamus (Guillery and Sherman, 2002a). But although a great deal of information has accumulated about the function of thalamic neurons conveying the qualities and receptive fields of sensory input, little is known about the characteristics and movement fields of thalamic neurons that monitor our actions. This chapter aims to draw on the recent experimental observations of ascending motor pathways passing through the thalamus in the hope of bringing the emerging understanding of these motor pathways closer to the extensive understanding of the sensory pathways.

The study begins with reviewing the concepts that have been developed for the ascending sensory 
pathways by Guillery and Sherman (Sherman and Guillery, 2001, 2002; Guillery and Sherman, 2002a,b; Guillery, 2003). Next it is investigated how the ascending motor information fits with their view of thalamocortical organization, particularly their concepts of drivers and modulators. Since these experiments explore the nature of signals at different levels in the ascending motor systems, the analysis concentrates on this functional level rather than on anatomical structure - on signals rather than synapses. In addition, the experimental observations pertain to the guidance of saccadic eye movements, so the discussion of the nature of ascending signals concentrates on visual and oculomotor functions.

To begin with the sensory side, Fig. 1, which is based on the review by Guillery and Sherman (Guillery and Sherman, 2002b), gives an outline of these ideas. Sensory information from the periphery passes through thalamic nuclei devoted to the sensory pathway: first order thalamic relays (Fig. 1, left). In addition to these first order nuclei, other thalamic nuclei that do not transmit such unmistakably ascending information have been referred to as higher order nuclei (Fig. 1, right). Instead of passing sensory

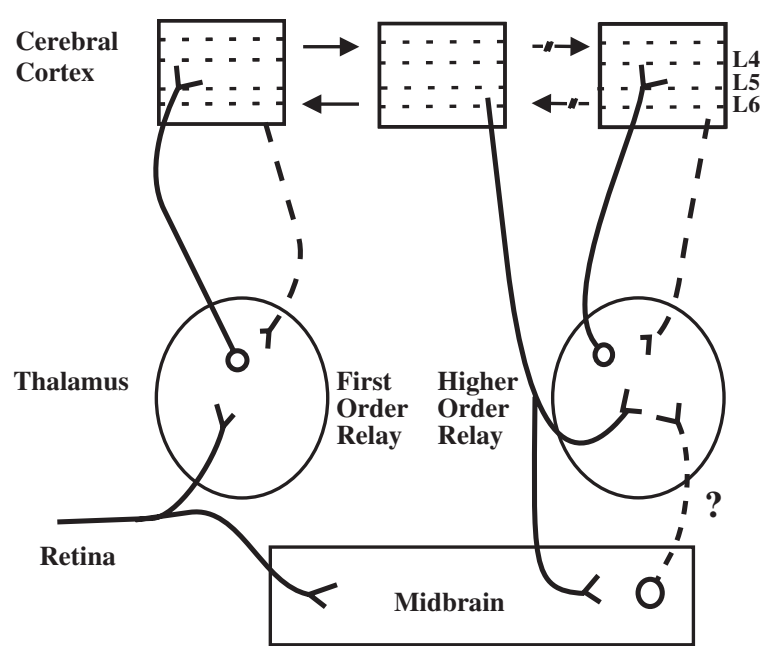

Fig. 1. Schematic representation of drivers and modulators and the problem of subcortical input. Solid lines represent drivers, dashed lines modulators, and the dotted line with the question mark represents the input considered in this article. See text of Introduction for description. Diagram after Guillery and Sherman (2002a). information up to cortex, these higher order nuclei are thought to convey information between cortical areas by transmitting signals from cortex to thalamus and then back to the cortex, providing a route for information flow between cortical areas that is independent of the direct corticocortical connections. In the visual system (on which the study concentrates), the lateral geniculate nucleus (LGN) is the first order nucleus, and other nuclei, particularly the medial dorsal nucleus (MD) and the pulvinar nuclei, are examples of higher order nuclei.

In addition to the source and destination of projections, what Sherman and Guillery established is that inputs to the thalamus can be distinguished on the basis of their anatomical and physiological characteristics. Inputs referred to as drivers are those that determine the receptive field properties of the relay neurons on which they impinge. More generally, these inputs carry the basic information on which the neuronal computations are based (Guillery and Sherman, 2002b). At an anatomical level the drivers frequently form a tight glomerular synaptic structure on the thalamic relay neuron. In the case of the visual system, the driver is the retinal input conveyed by the optic tract to the lateral geniculate nucleus, since the receptive fields of LGN neurons are dominated by signals from the retina, and the glomerular structure is striking in the LGN (drivers are the solid lines in Fig. 1). In the case of higher order nuclei, receptive field structure and synaptic organization led Guillery and Sherman to conclude that the input from cortical layer 5 is a driver input in such higher order thalamic nuclei as has recently been demonstrated in the pulvinar (Van Horn and Sherman, 2004).

In contrast to drivers, modulator inputs are those that modify but do not dominate the information content of the signals relayed by the thalamus. This modulation is present in both first and higher order nuclei; both the LGN and pulvinar, for example, receive such modulatory input from layer 6 of cortex (modulators are the dashed lines in Fig. 1). The study does not specifically consider modulators further although their interactions, particularly from the thalamic reticular nucleus (Montero, 1997, 2000; McAlonan et al., 2000; McAlonan and Wurtz, 2004), clearly influence the visual pathways. 
The organization shown in Fig. 1 represents an established view of the thalamic organization of sensory signals, including the outputs from layers 5 and 6 of cortex to the higher order nuclei. But inputs to higher order nuclei are not limited to the descending cortical inputs demonstrated by Sherman and Guillery; there are prominent inputs to these nuclei from subcortical areas as well. In the case of a first order nucleus, the LGN modulation of visual activity by eye movements has been recognized for many years and the effect on visual responses has recently been quantified (Ramcharan et al., 2001; Reppas et al., 2002). This modulation appears to arise from the brainstem (see discusion by Reppas et al., 2002). Since these subcortical inputs seem to alter the gain of the transmission through the LGN but not the fundamental organization of the thalamic neuronal receptive fields, these inputs have been regarded as modulators.

For higher order nuclei, such subcortical inputs have been equally well documented. Inputs from the superior colliculus (SC) to MD and the pulvinar (Benevento and Fallon, 1975; Harting et al., 1980; Stepniewska et al., 1999) are prominent examples considered in detail in this chapter. These subcortical inputs are represented by the line ascending from the midbrain to the higher order nucleus in Fig. 1 (dotted line, lower right). The unresolved question is, what is the nature of this subcortical input to the higher order nuclei? In the parlance of Guillery and Sherman, are these inputs drivers or modulators? Since it is known that the subcortical inputs to the first order thalamic relays that have been studied (such as the LGN) are modulators, there is reason to believe that this might be the case in general for first order nuclei, and that this may hold for higher order nuclei as well. There is no reason to doubt the modulatory role of subcortical input on first order relays, but there are a number of functional reasons to believe it might not be prudent to assign these inputs an exclusively modulatory role on higher order relays. The present thesis is that the projections from superior colliculus to thalamus are drivers too.

In this chapter a few observations on the pathway from the SC through the MD thalamus to the frontal eye field (FEF) region of prefrontal cortex are reviewed first. The observations on this pathway will be relevant to evaluating the functional role of subcortical inputs to a higher order thalamic nucleus. Both the characteristics of neuronal activity along the pathway and the finding that the signals conveyed by this pathway play a role in providing a corollary discharge are taken into consideration. The main arguments are listed next as to why these SC-MD projections are considered as drivers. Finally the study considers whether the shift of visual attention that is seen as a result of electrical stimulation of the $\mathrm{SC}$, the results of which most likely reach cortex through the pulvinar, is more consistent with a driver or modulator function. Taking these observations together, it is concluded that all of these ascending projections from the SC should be considered drivers rather than modulators of higher order thalamic nuclei. The point is that these inputs to the thalamus convey driving signals that encode imminent movements, used as corollary discharge, just as the driving inputs from the periphery encode recent sensory events.

\section{Signals conveyed by the SC-MD-FEF pathway}

The first step in determining the signal conveyed to a higher order nucleus from a subcortical area and then to the cerebral cortex is to specifically identify the neurons that relay the information. MD conveys information from multiple subcortical areas, including the superior colliculus, the cerebellar nuclei, and the basal ganglia, to wide regions of the frontal cortex. The seemingly impossible problem of determining which neurons in MD receive a given input and project to a given region of cortex can be solved by supplementing single neuron recordings with the classic physiological techniques of antidromic and orthodromic stimulation (Sommer and Wurtz, 2004a). From previous work, it is known that one of the visuomotor areas in frontal cortex, the FEF, is a main target of the SC-MD pathway (Lynch et al., 1994). MD neurons projecting into FEF could be identified by antidromically activating them from FEF (Fig. 2A). MD neurons receiving inputs from SC could be identified by orthodromically activating them from the SC (see Sommer and Wurtz, 2004a for limitations to the orthodromic method). Neurons that met both of these criteria were considered to be MD relay neurons, and the characteristics of the 


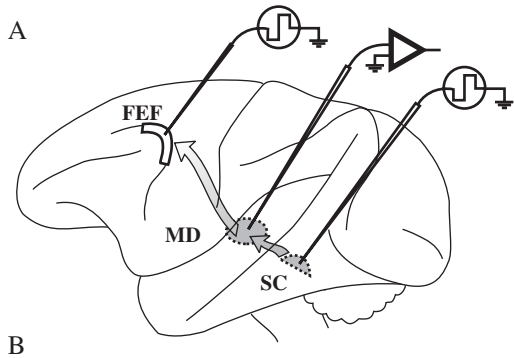

B

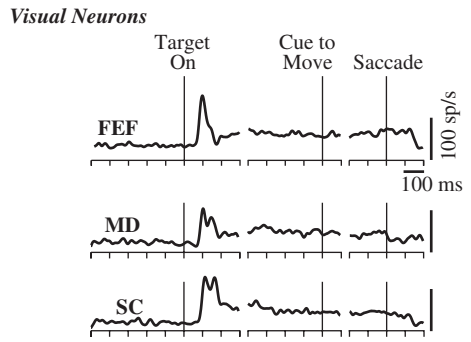

Visuomovement Neurons
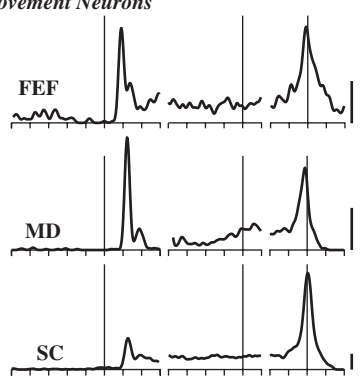

Movement Neurons

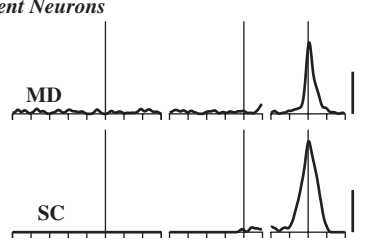

Fig. 2. Visual, delay, and movement activity of neurons in the SC-MD-FEF pathway. A. All MD neurons were identified as relay neurons by being antidromically activated from the FEF and orthodromically activated from the SC. B. Signals conveyed in the ascending pathway as determined using the delayed-saccade tasks. The monkey looked at a fixation spot and then a peripheral target appeared (Target On). The target remained on during an extended delay period, and disappearance of the fixation spot was the cue to make a saccade to the target location (Cue to Move). The records for Visual, Visuomovement, and Movement neurons are spike density functions (Gaussian width $10 \mathrm{~ms}$ ) showing the average firing rates of example FEF recipient, MD relay, and SC source neurons during the task. Data are aligned to target onset at left, to fixation spot offset (Cue to Move) in the middle, and to saccade onset at right. From Sommer and Wurtz (2004a). information conveyed through MD that is described is based on these identified relay neurons. In addition, neurons were identified in the SC that were the source of the input to MD by identifying SC neurons that were antidromically activated from $\mathrm{MD}$, and neurons were identified in FEF that were the recipients of input from SC through MD by orthodromically activating them from SC. A sample of neurons was thus obtained at each of the steps from SC to MD to $\mathrm{FEF}$, with each neuron in each sample shown to be a part of this pathway.

What signals do the neurons along this pathway convey? A delayed saccade task was used to determine whether the neurons responded to visual stimuli, whether they were active with saccades, and whether they had continuing delay activity between the visual and saccade related activity. The sequence of events in this delayed saccade task is indicated by labels at the top of Fig. 2B, and each section of Fig. 2B shows comparative examples of individual MD relay neurons, FEF recipient neurons, and SC source neurons. It was found that $87 \%$ of the MD neurons fell into three categories: some had only a phasic or tonic visual response but no presaccadic activity (Visual Neurons, Fig. 2B top), some had presaccadic activity but no visual response (Movement Neurons, Fig. 2B bottom), and others had both visual and presaccadic activity (Visuomovement Neurons, Fig. 2B middle). These same categories could be identified equally well in both FEF and SC (except for Movement Neurons, which were never found in the FEF recipient neuron sample). Figure $3 \mathrm{~A}$ shows the frequencies of these categories at each step of the pathway. A number of salient observations emerge from comparing the activity at different levels of the pathway:

(1) Nearly every neuron in the pathway was active in the oculomotor task, with only a small proportion at each stage showing no significant change in activity during the task (the "other" neurons). This indicates that many of the signals are seen in the pathway and thus one can draw conclusions with some confidence on the information the pathways conveys.

(2) The distributions of neuron types along the pathway may be compared. From SC to MD there was no significant change in the distribution of neuron types, consistent with the 
A

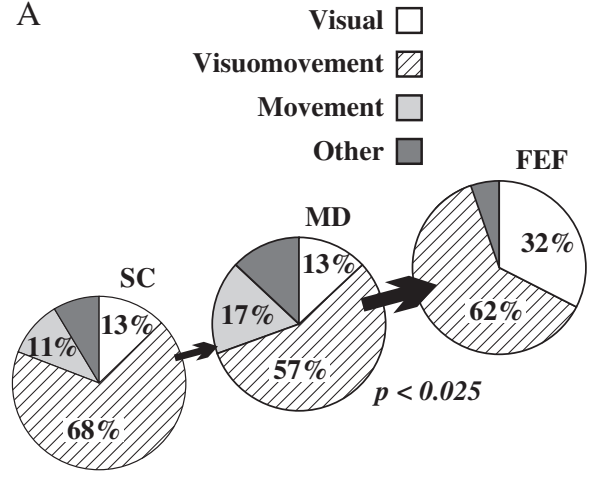

B

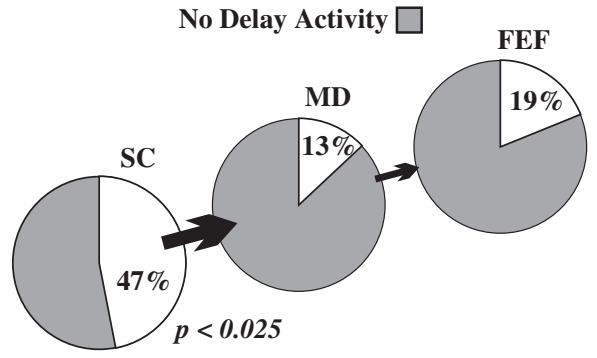

C

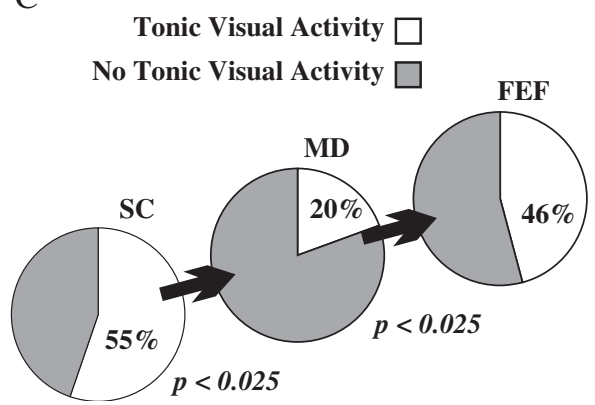

Fig. 3. Frequency of types of neurons at each stage in the ascending SC-MD-FEF pathway. A. Percentages of Visual, Visuomovement, and Movement neurons, B. Percentage of neurons having delay activity. C. Percentage of neurons having tonic visual activity. Bold arrows indicate significantly different distributions. The charts represent $47 \mathrm{SC}$ neurons, $46 \mathrm{MD}$ neurons, and 37 FEF neurons. From Sommer and Wurtz (2004a).

possibility that the controlling input to MD was from the superior colliculus. From MD to FEF the distribution changed significantly: the FEF distribution had much more visual activity than would be expected from its MD input. In addition, when this visual response occurred, it was found that it was nearly simultaneous in MD and the SC but about $15 \mathrm{~ms}$ earlier in the FEF (Fig. 4). Thus it seems likely that the visual input from MD is added on to an existing visual response in FEF that presumably results from extrastriate input.

(3) As is evident in Fig. 2B, many of the neurons also had delay activity, a signal occurring after target disappearance and before saccade initiation, which may be involved in higher level cognitive functions such as target selection or working memory (Goldman-Rakic, 1995; Fuster, 1997). Delay activity was present in all of the visuomovement neurons in Fig. 2B, for example. Figure 3B shows that from $\mathrm{SC}$ to $\mathrm{MD}$, the proportion of neurons with delay activity dropped significantly, while from MD to FEF it did not change. Thus the connection from SC to MD seemed to act like a high-pass filter; the connection seemed to let bursts of activity through more readily than sustained activity. Consistent with this possibility, it was also found that the proportion of neurons carrying another sustained signal, tonic visual activity, decreased from SC to MD (Fig. 3C). In contrast, the proportion of tonic visual signals increased from MD to FEF, which also provides added evidence that the FEF recipient neurons are generally more visual than expected from their MD input, consistent with the suggestion that the FEF recipient neurons receive additional visual signals from elsewhere such as from extrastriate cortex. But the main point is that delay activity is largely suppressed at the SC-MD synapse.

Figure 5 summarizes the signals conveyed in the SC-MD-FEF pathway. Diverse signals, from visual to delay to motor, are sent up to thalamus from the $\mathrm{SC}$ and most of them continue on to the FEF except for highly damped delay activity (Fig. 5A). The signals that enter the FEF from the SC via MD must have different influences (Fig. 5B): the visual activity arrives too late to directly cause visual responses in FEF and the delay activity seems too little in quantity to be of any substantial import, but 
Visual Burst

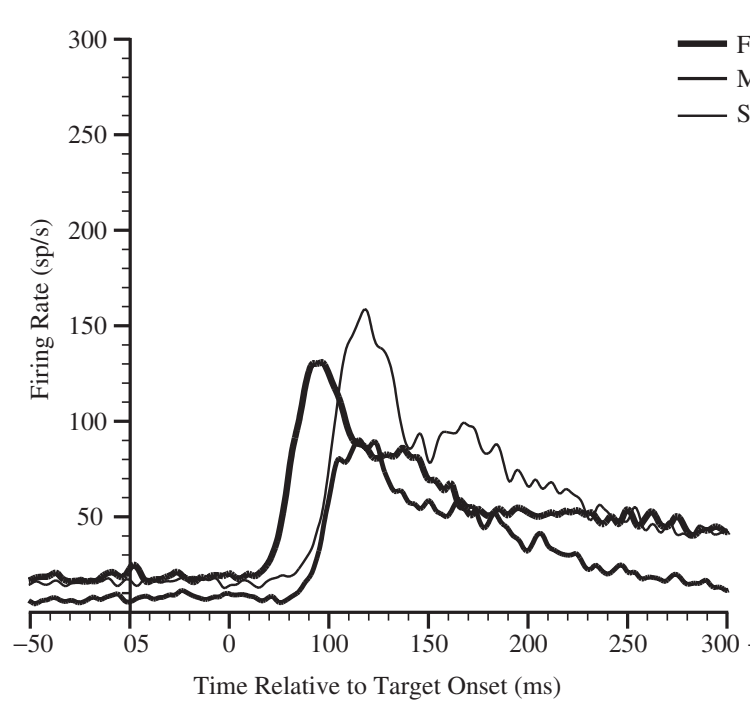

Saccadic Burst

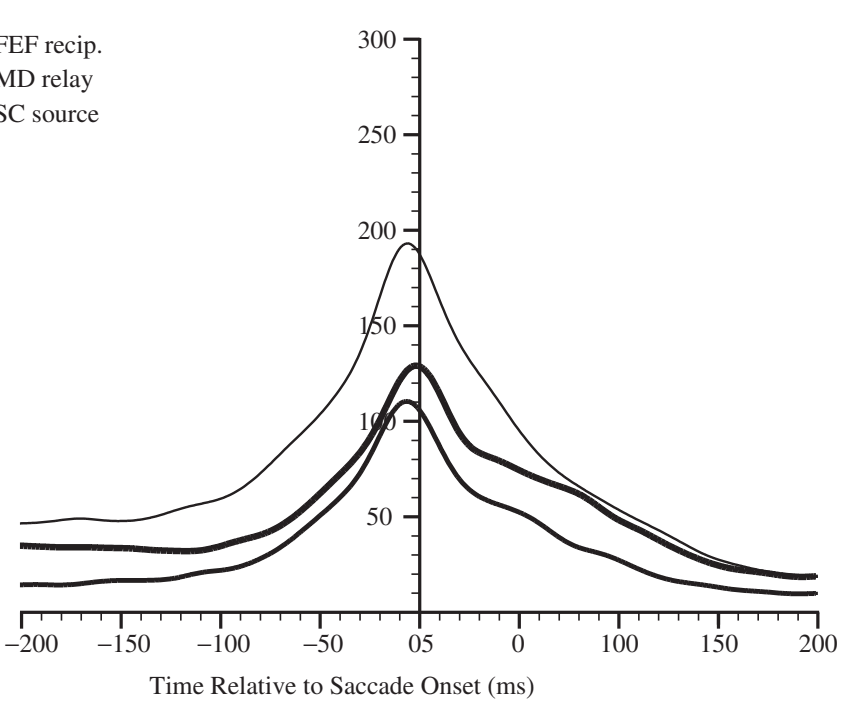

Fig. 4. Timing of signals along the SC-MD-FEF pathway. Mean activity in the SC, MD, and FEF samples are aligned to target onset (left) to show the visual burst and to saccade onset (right) to show the presaccadic burst. From Sommer and Wurtz (2004a).

the motor-related activity seems just right to exert an important impact, as it is very common, strong in magnitude, and arrives just prior to a saccade.

\section{A corollary discharge function for the SC-MD-FEF pathway}

Activity in the SC-MD-FEF pathway could provide a variety of types of information and probably does so not only for the visual tasks that have been investigated but for other tasks as well. The next task after determining the types of signals conveyed through MD was to explore what the function of these signals might be (Sommer and Wurtz, 2004b). The obvious possibility given the visual responses and the presaccadic activity is that these neurons are providing a signal for the guidance of saccades. This was tested in the same way that this question has been investigated previously in the SC and the FEF, by inactivating the neurons and then testing the effect on the monkey's ability to make saccades to visual targets. MD neurons were inactivated using muscimol, a GABA agonist, injected at sites where the MD relay neurons were located. Figure 6 shows that such inactivation did not significantly alter the accuracy of the saccades either in individual examples (Fig. 6A) or in the entire study (Fig. 6B). In contrast, previous experiments have shown that such saccades are clearly altered after such inactivation of both the SC source of this pathway and its FEF target. What is conveyed upward by MD must be contributing something other than the essential information for saccade generation.

Another possibility is that the activity is not conveying information for generating the movement but for keeping track of it, that is, it is carrying a corollary discharge (efference copy) of the intended movement. This could also be tested by inactivating MD while using a task designed to reveal a change in behavior if the corollary discharge for saccadic eye movement were impaired. In this double saccade task the monkey fixated a spot, the spot disappeared, and two targets briefly appeared in sequence (Fig. 7A). To receive a reward the monkey had to make two sequential saccades to the locations of the targets. The targets were brief flashes so that both had disappeared before the first saccade began, and the monkey was in the dark with no other visual cues present. 

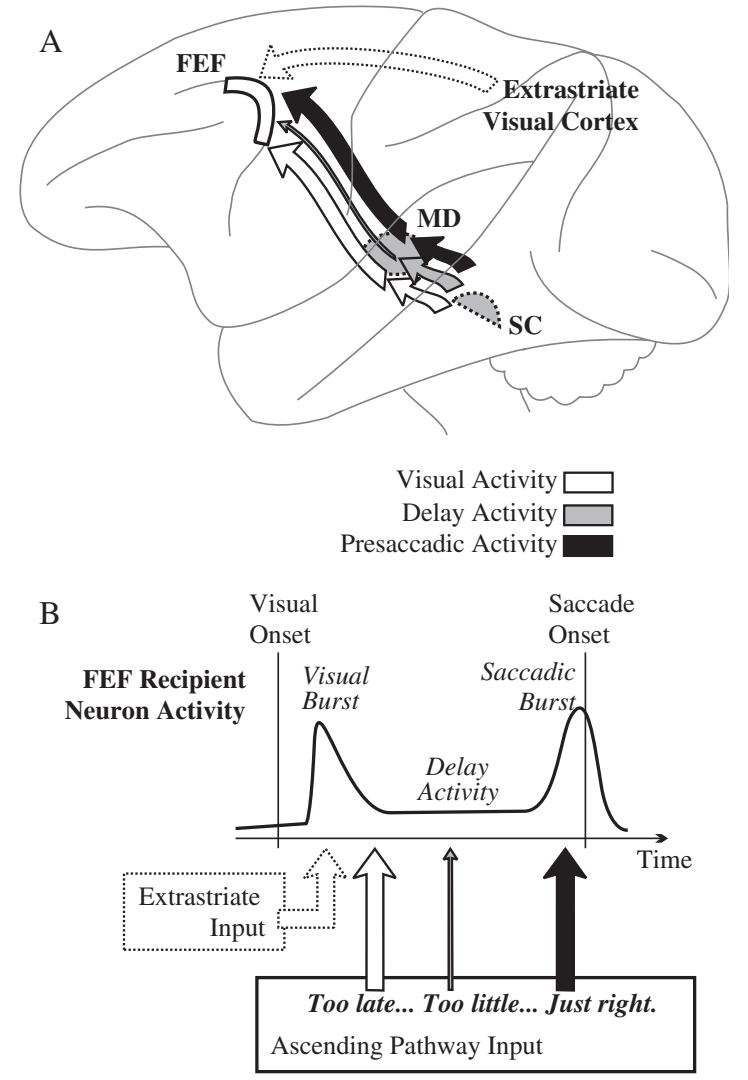

Fig. 5. Summary of the signal content in the SC-MD-FEF pathway. A. Visual, delay, and pre-saccadic activity are all sent from SC to MD, and all three continue to FEF except that the amount of delay activity is severely reduced. At the FEF, neurons receiving this ascending input also seem to receive extra visual input, presumably from extrastriate cortex. B. The relative contribution of SC input to the FEF. MD to FEF visual signals from the ascending pathway arrive too late to cause the FEF visual burst, which is probably initiated by extrastriate input. It appears that too little delay activity survives through the pathway to be of major importance. However, the presaccadic activity seems just right; it travels unhindered through the pathway and arrives precisely at the appropriate time to contribute to saccadic bursts in the FEF neurons. From Sommer and Wurtz (2004a).

The location of the target pairs was randomized so that the monkey could not predict which pattern of targets would occur on any given trial. The point of the task is this: in order to make the second saccade straight up, the monkey has to know that its eye moved and where it moved to during the first saccade. If it did not know this, it should make a diagonal saccade up and to the right as if the eye were still looking at the original target point. The constant internal monitoring of saccadic behavior needed for this task must result from an extraretinal signal which is hypothesized as from the corollary discharge information conveyed by the SC-MD-FEF. The reason for this is that proprioception is unlikely to provide the eye position information; this has been discussed previously (Sommer and Wurtz, 2004b). Note that the fact that neurons in the pathway increase activity before the saccade (Fig. 4, right) indicates that activity cannot be proprioceptive since the eye has not yet begun to move.

Figure 7B shows saccades during an MD inactivation in the double step task. The monkey had to make a rightward saccade and then an upward saccade in response to two flashed targets. Figure 7B, top shows all the saccades made before MD was inactivated. Most second saccades were made appropriately upward. During MD inactivation (Fig. 7B, bottom), the monkey's behavior was nearly the same except that the second, upward saccades seemed to be tilted toward the upper right, in the direction expected if the monkey did not know the first saccade had been made. In Fig. 7C all the trials in this session are summarized by showing the means and SDs of the initial fixations, first saccade endpoints, and second saccade endpoints before and during inactivation. The most obvious effect of the inactivation seemed to be a roughly horizontal shift in second saccade endpoints, and this shift was significant. In contrast, neither the initial fixations nor the first saccade endpoints shifted in either the horizontal or vertical direction. This result was confirmed across multiple injections in two monkeys (Sommer and Wurtz, 2004b).

If the corollary discharge occurs with each saccade, it would also be expected to see deficits on a trial-bytrial basis when the corollary discharge is impaired, and this did happen. That is, not only the mean accuracy was affected but also the precision, trial-by-trial, was impaired. Figure 8A, left, shows the trial-by-trial variation in second saccade vectors that resulted from the fact that the first rightward horizontal saccade had slight variability in amplitude from trial to trial for which the second saccade compensated. In Fig. 8A, the second saccades have been ordered according to the end of the first horizontal saccade 


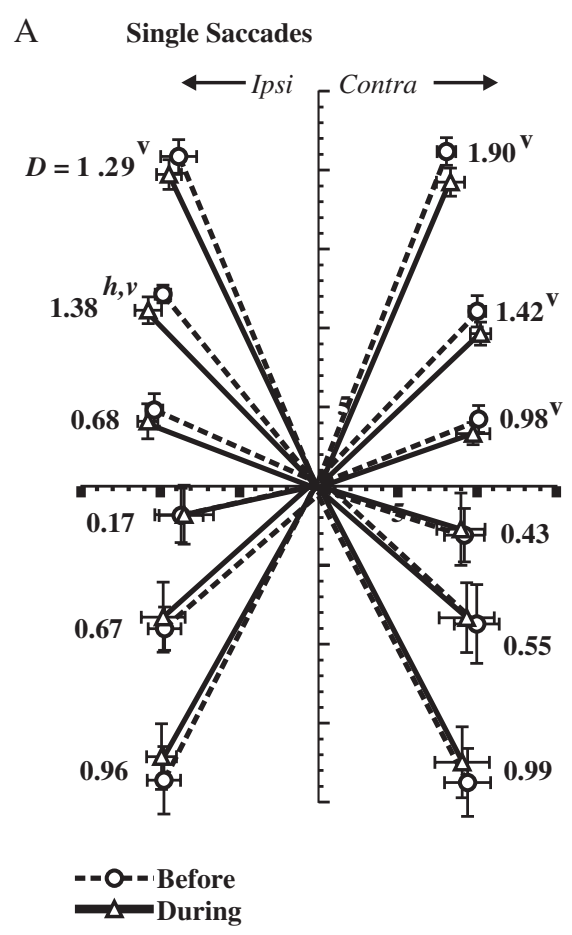

B

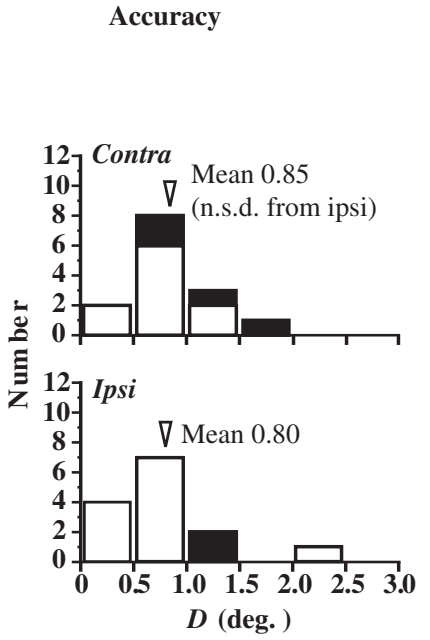

Significant shift either horizontally

or vertically $(p<0.025)$

Fig. 6. Lack of impairment for saccades made to single targets during MD inactivation. A. Summary of single saccades made before and during one example inactivation. The panels represent 12 pairs of before-during saccadic endpoints in each direction (contraversive and ipsiversive), and the difference (D) in the horizontal (h) and vertical (v) directions is indicated in degrees. B. Histograms showing how accuracy was affected by inactivation in all the experiments. There were negligible effects and no significant difference in contraversive vs. ipsiversive directions. See Sommer and Wurtz (2004b) for further experimental details.

(the shortest first saccade at the top, the longest at the bottom). Before the injection (Fig. 8A, left) the second saccade compensated for the variation in first saccade amplitude: shorter first saccades (top) tended to be followed by a saccade that went further to the right to compensate for the first saccade falling short; longer saccades (bottom) tended to be followed by a saccade that was actually directed backward to the left to compensate for the first saccade being too long. Thus the monkey was quite deft at precisely adjusting the second saccade direction, from which it is inferred that it had information about where the first saccade ended with each saccade made.

In contrast, during the inactivation of MD (Fig. $8 \mathrm{~A}$, right) there was much less compensation: all of the second saccades were directed toward the right regardless of whether the first saccade was relatively short or long on a particular trial. Figure $8 \mathrm{~B}$ shows this result quantitatively by comparing the directions $(\theta)$ of ideal second saccades that would be expected from perfect compensation (Sommer and Wurtz, 2004b) with those of the actual second saccades that were observed. Before inactivation, when corollary discharge was present (bold circles and line), a correlation was noticed between the ideal and observed data, and the linear regression slope was near one. During the inactivation (thin triangles and line) the relationship was reduced: the correlation became insignificant. This result was representative of all the inactivations. In sum, monkeys normally adjusted their second saccade directions from trial to trial to compensate for slight fluctuations in first saccades, and this was disrupted by inactivation. It is concluded, therefore, that corollary discharge is normally precise and that MD inactivation impairs this precision. 


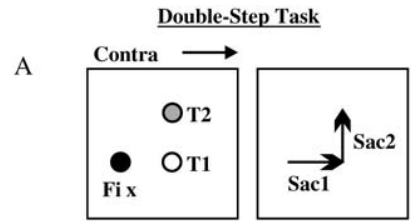

Individual Trials
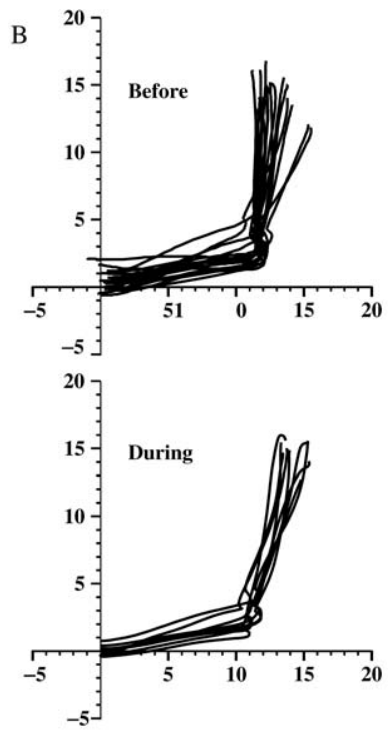

Mean Vector Direction

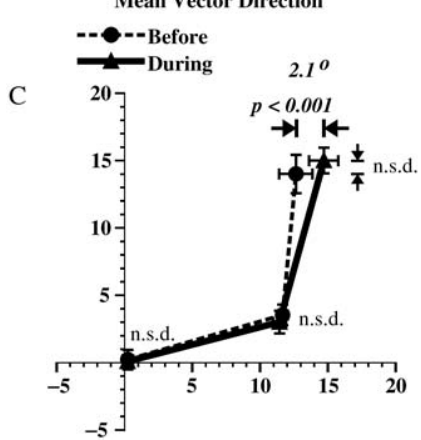

Fig. 7. Loss of corollary discharge during MD inactivation. A. Spatial aspects of the double step task. Monkeys initially looked at a fixation spot (Fix) and then two targets (T1 and T2) appeared sequentially in the periphery. The task was to make two saccades (Sac1 and Sac2) to the locations of the extinguished targets. B. Example data from the double-step task before and during MD inactivation. Superimposed are saccades from correct trials. C. Summary of the correct trials before and during inactivation, showing the means and SDs of initial fixation locations, first saccade endpoints, and second saccade endpoints. The only significant change during inactivation was a contraversive shift in second saccade endpoints, indicating a loss of corollary discharge accuracy. n.s.d., not significantly different. From Sommer and Wurtz (2004b).

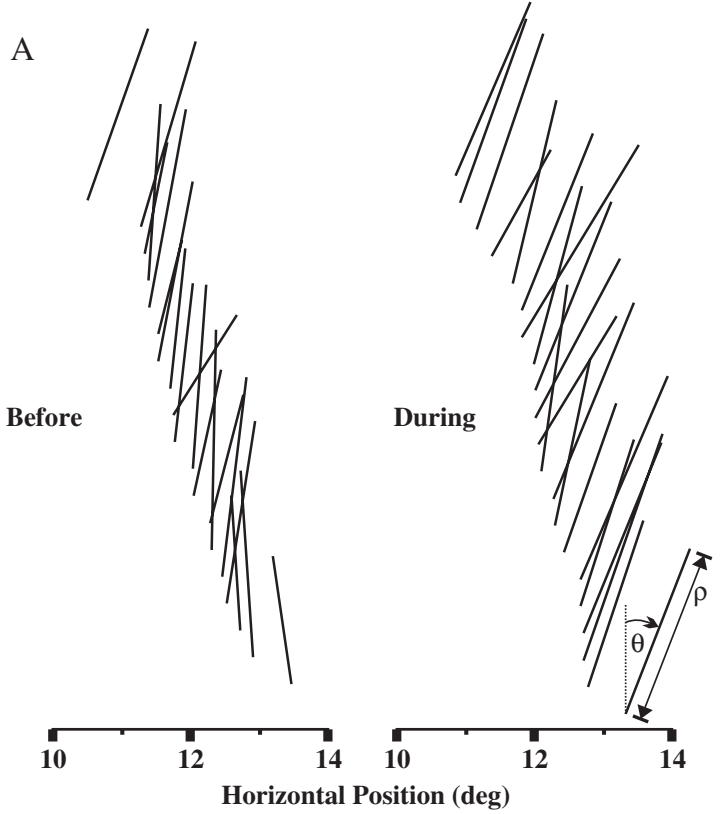

B

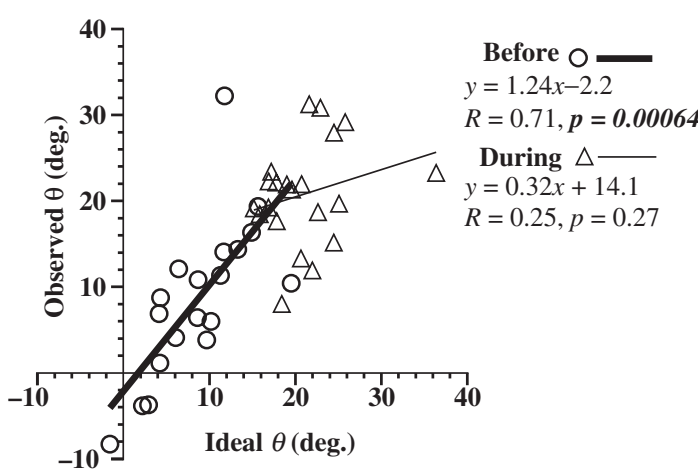

Fig. 8. Loss of corollary discharge precision during MD inactivation: a trial-by-trial analysis. A. Individual second saccades from an experiment with targets positioned as shown in Fig. 7A. Second saccades before inactivation are shown at left and during inactivation at right. Each saccade is represented by a vector connecting its initial and final position with a line, and the vectors are ordered by the starting position of the second saccade which indicates the ending position of the first saccade. B. How well the monkey adjusted its second saccade directions to account for trial-by-trial fluctuations in its first saccades. The observed $\theta$ for each second saccade (from the vectors in $\mathrm{A}$ ) is plotted against the ideal $\theta$ (if corollary discharge was perfect). Before inactivation there was a direct correlation between observed $\theta$ and ideal $\theta$ with a linear regression slope near unity (1.24), but during inactivation the correlation was not significant and the slope was only 0.32 . From Sommer and Wurtz (2004b). 


\section{SC As a driver input to MD}

Thus far recent work on the signals that are conveyed through the SC-MD-FEF pathway were reviewed. Inspired by anatomical evidence for such a disynaptic route (Benevento and Fallon, 1975; Harting et al., 1980; Lynch et al., 1994) neurons were physiologically identified throughout the pathway and inactivated at the thalamic level. It was concluded that the pathway carries corollary discharge information. These conclusions presuppose that the SC drives MD relay neurons, but what direct evidence is there for this presumption? Is the SC really a driver of MD relay neurons, as the functional evidence suggests, or is it a modulator? The present observations, taken as a whole, make a strong case for the SC input as being a driver onto $\mathrm{MD}$, but we readily concede that no one piece of evidence is conclusive. For example, perhaps inactivating MD caused corollary discharge deficits because the MD relay neurons carry corollary discharge as supplied not by SC ascending input, but by collaterals of FEF layer 5 descending projections as depicted in Fig. 1. Fundamentally, therefore, skeptics could still protest that it remains ambiguous as to whether MD relay neuron activity is driven by ascending SC input or by descending FEF input. In this section the seemingly most compelling four arguments for the thesis that the $\mathrm{SC}$ provides a driver input to MD neurons have been summarized.

The first argument is based on the structure and speed of the SC-MD connection. The speed is necessarily related to the structure, which is why these points are combined. While direct evidence is not available concerning the anatomical arrangement of synapses on MD neurons comparable to that in other thalamic regions (e.g., the pulvinar, Guillery, 1995; Reichova and Sherman, 2004), there are a few observations that suggest that the SC to MD synapses are strong and secure, which make them reasonable candidates for drivers. First of all, it was found that single pulse stimulation in the SC activated MD neurons with short latency (median $1.4 \mathrm{~ms}$, including a presumed synaptic delay of only $0.57 \mathrm{~ms}$ ) and required normal current thresholds for this type of study (mean $264 \mu \mathrm{A}$ ). While ultrastructural anatomy of MD is not yet available in the monkey, the anatomy of synapses in the rat have been studied by Kuroda and Price (1991). They found that the "collicular boutons tend to be larger and distribute to more proximal parts of the dendrites than those from the prefrontal cortex" and that the SC inputs made asymmetric, excitatory synapses onto the thalamic neurons. While the location of these synapses has to be evaluated in comparison to other structures, such connections close to the soma have also been reported by Kelly et al. (2003) in the collicular projection to the pulvinar and lateral posterior nuclei of the cat. Both the physiological and anatomical observations are consistent with the strong driving of MD by the SC and suggests that SC inputs probably have more influence on the MD relay neurons than do cortical inputs.

Second, the signals conveyed by SC neurons projecting up to MD, and MD relay neurons themselves, are remarkably similar. This is true not only in terms of the proportion of cell classes in each population (Fig. 3A), but also in terms of their visual- and saccade-related activity profiles (Fig. 4). In essence, the MD neuronal activity simply looks like a slightly muffled version of the SC activity; this damping is not surprising and just implies that not every single spike emanating from SC causes a spike in $\mathrm{MD}$ - the synapses are strong and fast but not perfect. Moreover, as noted above, the SC-MD synapse seems to have a high-pass filter characteristic as seen for other drivers onto thalamus (e.g., in the retinogeniculate projection, discussed by Sommer and Wurtz, 2004a). It should be noted that the subpopulation of FEF neurons projecting onto the MD relay neurons might also have activity profiles similar to the MD neurons. This subpopulation of FEF neurons has not yet been studied, however.

The third point is based on an interesting negative result: thus far there has been very little evidence for a strong projection of FEF onto the MD relay neurons. Present observations on this matter are limited but seem to suggest that the FEF influence on MD relay neurons is very weak. Figure 9 shows one example. Recording was done from an MD neuron while applying a brief pulse of stimulation in the FEF. The purpose was to see if the neuron could be activated antidromically the neuron which would demonstrate that it projected into the FEF (Fig. 9A, "Anti"). But the stimulation could also orthodromically activate the MD neuron if it were receiving input from the FEF (Fig. 9A, "Ortho"), and indeed this 

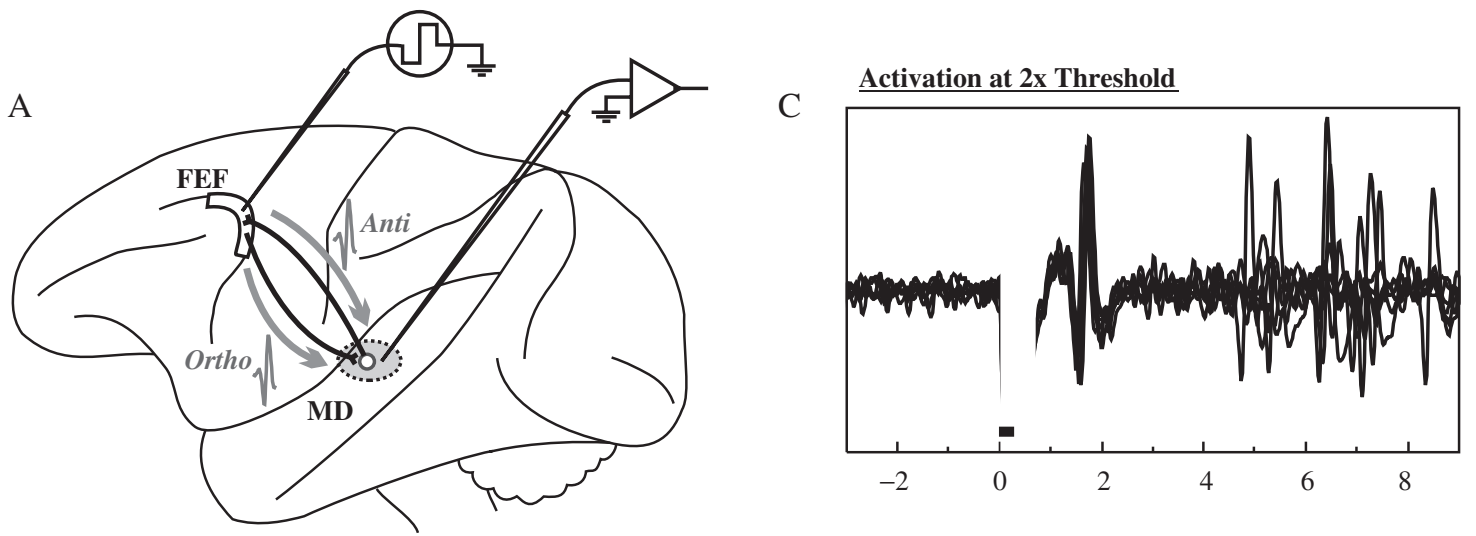

Activation at Threshold

B

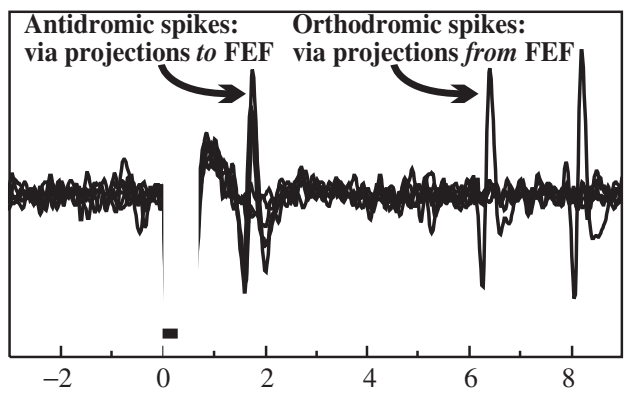

\section{Collision Test at 2x Threshold}

$\mathrm{D}$

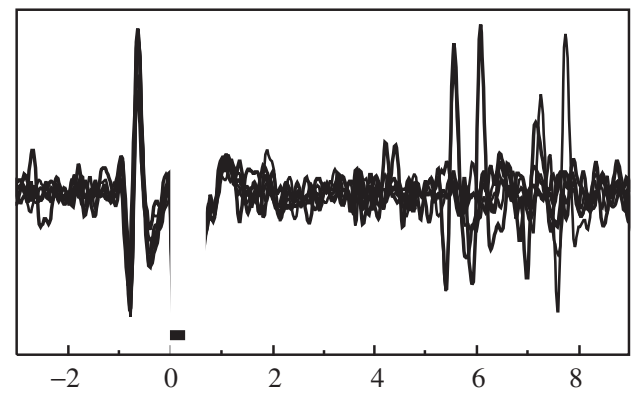

Time relative to onset of FEF stimulation (ms)

Fig. 9. Evidence for slow FEF input onto MD relay neurons. A. While recording from an MD neuron, a brief pulse of current was applied to the FEF. This evoked an action potential (depicted in gray) that could travel either backwards in the ascending axon of the MD neuron (i.e., antidromically, Anti), or forwards in the descending axons of FEF neurons (i.e., orthodromically, Ortho). B. Action potentials from an example MD relay neuron while stimulating at threshold. Results of several trials are superimposed; stimulation in FEF started at time 0 and its duration is indicated by the short bar above the abscissa. Because stimulation was at threshold, it evokes spikes in the neuron on about half of trials. As labeled, antidromic spikes occur early (about $1.2 \mathrm{~ms}$ latency) and orthodromic spikes late (about 6-8 ms latency). C. Same experiment but using twice threshold current, and the early antidromic and late orthodromic spikes are clearly seen to be caused by the stimulation. D. Collision test at twice threshold. Stimulation is synchronized to occur just after the initiation of a spontaneous action potential of the neuron (occurring at about $-1 \mathrm{~ms}$ ). This caused the annihilation of the early cluster of spikes (compare the waveforms in the range 1-2 ms between this panel and panel C), showing that they were antidromically activated, but it did not annihilate the later spikes, showing that they were orthodromically activated. For review of the collision test, see Lemon (1984).

occasionally happened (in about $10 \%$ of MD relay neurons). Figure 9B shows antidromic and orthodromic activation on a single MD neuron at the threshold current for antidromic activation $(55 \mu \mathrm{A})$. An antidromic spike occurred in half of the trials, and a few later spikes also occurred. By increasing the current (Fig. 9C) it became obvious that the later spikes were being caused by the FEF stimulation. Application of the collision test (Fig. 9D) proved that the early, highly stable spikes were antidromic (they disappeared) while the later ones were orthodromic (they remained). This neuron, therefore, both projected to the FEF and received projections from it. The long and variable latencies of the orthodromic activation $(\sim 4.5-8 \mathrm{~ms})$ indicated that the influence of the FEF on the MD thalamic neuron was rather weak and inconsistent. Moreover, attributing this activation to a monosynaptic projection from the FEF has been generous, as it easily could have been due to polysynaptic pathways. In sum, the influence of FEF 
A

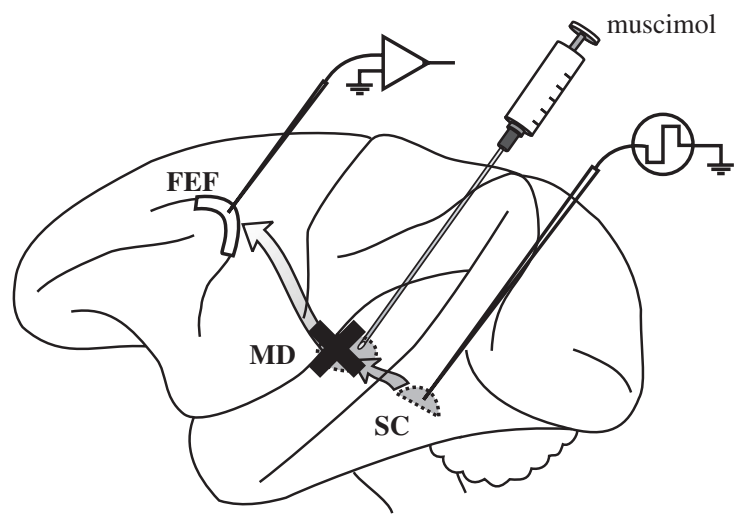

B

Spikes evoked in FEF neurons from stimulation of SC

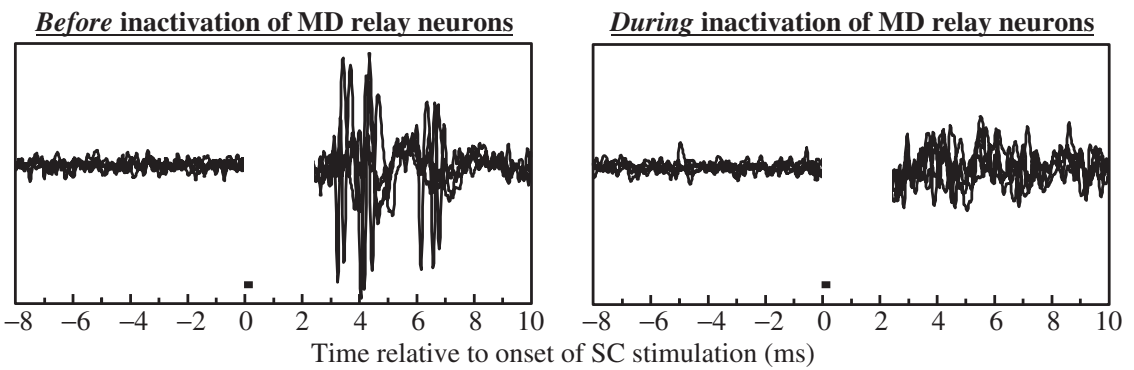

Fig. 10. Blockade of spike transmission from SC to FEF using MD inactivation. A. FEF neurons were recorded while brief pulses of stimulation were applied to the SC. B. Activity in an FEF neuron evoked by stimulation of the SC. Left: Before inactivation, SC stimulation (starting at time 0 for the duration indicated by the bar) caused a volley of spikes in the FEF. Right: During inactivation, the volley was severely reduced, indicating that the driving input of SC onto FEF was dependent on MD relay neurons.

on MD relay neurons would appear to be very limited and presumably modulatory according to these data. In contrast, as noted above, identical stimulation in the SC causes strong, short-latency (median $1.4 \mathrm{~ms}$ ) orthodromic activation of MD relay neurons, consistent with its being a driver input.

One caveat, however, is that the layer 5 projections from other cortical areas could also provide a driver input onto the MD relay neurons, but evaluating this from the present experiments is not possible because areas beyond the FEF were not stimulated. There has been no reason to reject the possibility of multiple driver inputs to the MD relay neurons.

The fourth and final point indicating a driving influence of SC onto MD is derived from the recent experiments (yet unpublished) using recording, inactivation, and stimulation simultaneously to determine the contribution of SC input through MD on FEF activity (Fig. 10A). We recorded from FEF neurons and attempted to drive each one with SC stimulation. When an FEF neuron that was orthodromically activated from the SC and thus presumably was driven through the SC-MD-FEF pathway was found, this presumption was tested explicitly by reversibly inactivating the MD relay neurons with muscimol. A typical result is shown in Fig. 10B. Before inactivation (Fig. 10B, left) stimulation in the SC caused a strong volley of action potentials in the FEF neuron (consisting of a shorter latency, larger amplitude spike and a second, later, smaller spike). Other orthodromic spikes were present in the background activity but are obscured by the large spikes. During inactivation of the MD relay neurons (Fig. 10B, right), using $0.9 \mu \mathrm{L}$ of $5 \mu \mathrm{g} / \mu \mathrm{L}$ muscimol, the SC stimulation failed to activate the two (larger and smaller) FEF neurons. Some background 
orthodromic activity was still present, but it seems likely that with a larger injection in MD this would have disappeared too. The point made by this experiment is that SC activation drives FEF neurons, and this driving is critically dependent on the activity of MD relay neurons; hence, activity in the SC must be driving MD relay neurons.

\section{SC and visual spatial attention}

In other recent experiments, another likely SC input onto the cerebral cortex has been identified, one related to the enhancement of visual processing through shifts of attention. The goal was to test the idea that common subcortical mechanisms underlie both the generation of saccades to one part of the visual field and shifts of attention to that same part of the visual field - a motor theory of attention (Rizzolatti, 1983; Sheliga et al., 1994; Moore et al., 2003). It was hypothesized that these two functions do not diverge until a point in the neuronal circuitry close to the actual motor neurons. Consequently, the well-known visuomotor map in the intermediate layers of the SC was targeted (Robinson, 1972), assuming that the point of divergence occurs after the SC. In addition to the increase in activity just preceding a saccade that was emphasized with respect to the SC-MD-FEF pathway, many of the neurons in the intermediate layers of the SC also have delay activity related to gradual selection of the saccade to be made (Glimcher and Sparks, 1992; Dorris and Munoz, 1995; Basso and Wurtz, 1998; Bell et al., 2004). Such selection-related activity occurs at the same time that neuronal activity in visual areas of the cortex is enhanced during attentional tasks (Reynolds and Desimone, 1999; Ghose and Maunsell, 2002). This SC selection-related activity is modulated when the monkey attends to a region of the visual field (Kustov and Robinson, 1996), and this modulation occurs only with a spatial cue for that region (Ignashchenkova et al., 2004). The logic then is that the delay activity of these SC neurons might be directed not only to preparing for a saccade to one part of the visual field but also to providing a spatial attention signal to cortex that modulates the activity of visual cortical neurons related to the same part of the visual field.
Since it was vital to the experiment to effectively manipulate the allocation of visual attention, we wanted to use a visual task that is strongly affected by visual attention. The task we chose was change blindness. Change blindness is the failure to see large changes in a visual scene that occur at the same time as global visual transients, such as the naturally occurring blurring caused by rapid or saccadic eye movements between fixations, or by brief blanks interposed between successive visual scenes presented during continued fixation. Attention shifts to the site of the change counter this "blindness" by improving both detection of the change and reaction time to the change. We developed a change blindness paradigm for visual motion and then showed that presenting an attentional cue diminished the blindness in monkeys as well as in humans. In this change blindness task (Fig. 11A), the subjects began each trial by fixating on a spot in the center of the screen in front of them and then three patches of random dot motion appeared: one target and two distractors (for details see Cavanaugh and Wurtz, 2005). On $65 \%$ of the trials, the direction of motion in the target patch changed, and the subject indicated such a change by making a saccade to the target if it changed, and continued fixating if the direction did not change. The direction of motion in the distractor patches never changed, and the location of the target patch was randomized among trials. On half the trials, before the patches appeared, a visual cue indicated which patch was the target while on the other half of the trials there was no such cue. To induce change blindness on cued and non-cued trials, a visual transient was initiated just when the direction of dot motion in the target might change. To measure the attentional cue's influence on change blindness the subject's performance on trials without a cue was compared with performance on trials with a cue. As expected, it was found that introducing the blank made the change in the direction of motion hard to see, thus demonstrating for the first time change blindness in monkeys as well as humans. It was also found that shifting attention by providing a visual cue increased the detection of the change in the target and decreased the reaction time for indicating the location of change - the two classic measures of visual spatial attention. 
A

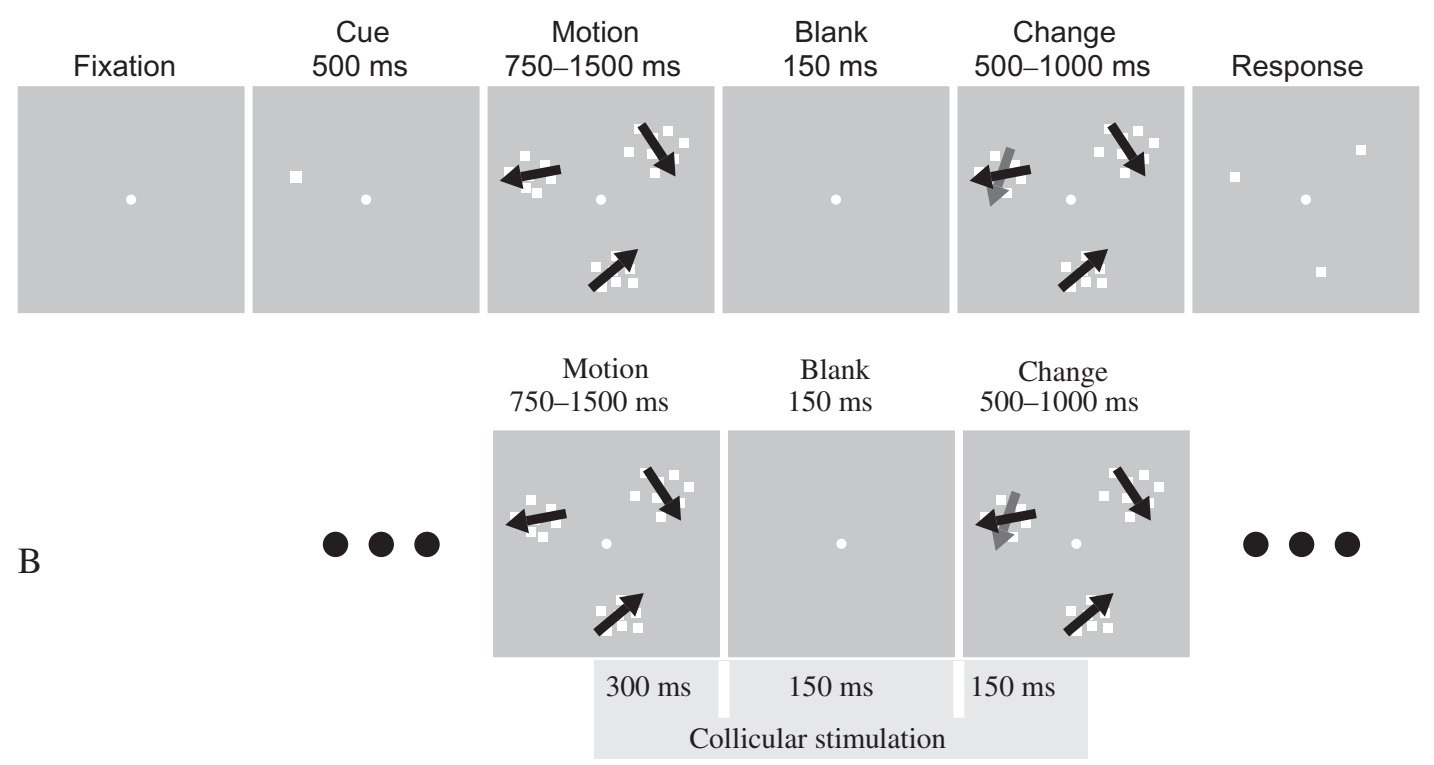

C

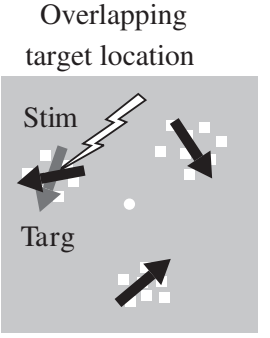

Non-overlapping

target location

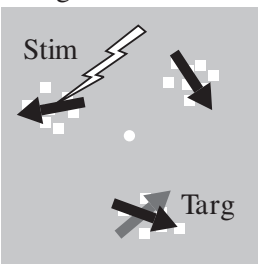

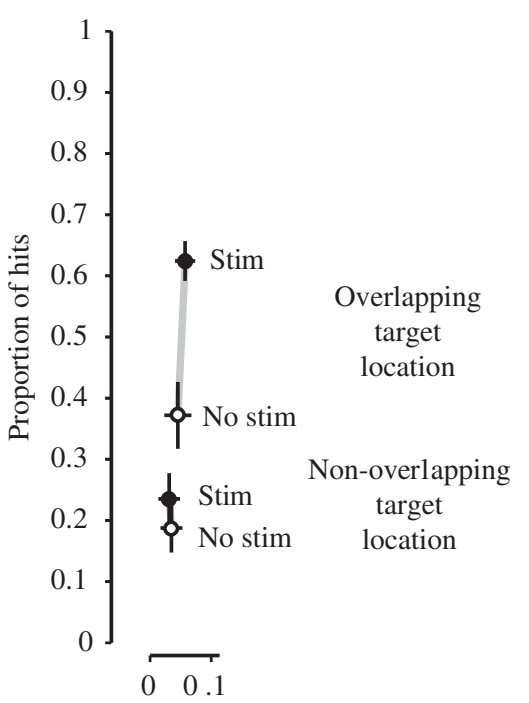

Proportion of

false positives

Fig. 11. Superior colliculus stimulation alters attention in a change blindness task. A. Sequence of stimuli presented in the behavioral change blindness task for those trials in which a cue was given. B. Time course of sub-threshold collicular stimulation. Stimulation began $300 \mathrm{~ms}$ before the blank period, and continued for $600 \mathrm{~ms}$, ending $150 \mathrm{~ms}$ after the patches reappeared. C. Sample results from an SC stimulation experiment showing the difference when stimulation was given (closed symbols) and not given (open symbols). The graph shows sample results from a single stimulation site, with the ordinate and abscissa indicating proportions of hits and false positives, respectively. In overlapping experiments, stimulation occurred when the target patch spatially overlapped the visual field location of the collicular stimulation site. In non-overlapping experiments, stimulation occurred when one of the distractors spatially overlapped the collicular stimulation site. When the target overlapped the site of collicular stimulation, the proportion of hits increased greatly, while there was little change in the occurrence of false positives to this location. In the non-overlapping experiment, neither hits nor false positives changed significantly. From Cavanaugh and Wurtz (2005). 
The experiment was then changed for the monkeys. Instead of providing a visual cue to indicate target location, the SC was electrically stimulated during the period when the change in direction might be occurring (Fig. 11B). After first identifying the part of the visual field to which the SC was related by evoking saccades with electrical stimulation, the stimulation frequency was reduced until it was too weak to elicit an eye movement. This low level stimulation was found to produce results compatible with a shift of attention - increased detection of the change and a faster reaction time. In the example shown in Fig. 11C, SC stimulation significantly increased the proportion of hits $(p=0.0001)$ when the target patch spatially overlapped the stimulation site. SC stimulation also reduced the reaction time for correct responses on these trials.

A separate set of trials was run to determine whether the increase in hits from stimulation resulted from some general effect of stimulation, such as arousal. In this set of trials, the SC was stimulated only when the target was in the opposite visual hemifield and did not overlap the stimulation site. In the example experiment (Fig. 11C) for this nonoverlapping case, no significant increase in hit rate was observed $(p=0.44)$.

Note that for this example stimulation site, in neither the overlapping nor the non-overlapping case did the false positive rate change significantly $(p>0.34)$. If $\mathrm{SC}$ stimulation simply caused more saccades, there would be more false positives to the target (incorrect saccades) as well as hits (correct saccades). The increase in just the correct saccadic responses suggests that stimulating the $\mathrm{SC}$ countered change blindness in a spatially selective manner, akin to shifting covert attention with a visual cue.

This effect was repeated over a number of SC stimulation sites and a modulation of performance was found to varying degrees. The mean increase in hit rate across overlapping experiments was significant $(9.2 \%, p<0.0001)$ as was the reduction in mean reaction time $(-14.9 \mathrm{~ms}, p=0.0001)$. Thus the observed change in the animal's performance met both criteria used to determine a shift of visual attention: a spatially selective increase in hits, and a reduction in reaction time. These results provide support for the motor theory of attention, specifically the hypothesis that the SC activity preceding the generation of saccadic eye movements to one part of the visual field also contributes a spatially selective attentional input for the enhanced visual processing seen in visual cortical areas.

What is the relevance of this for the present consideration of the nature of subcortical inputs to the thalamus? Unlike the pathway which has been identified from SC to MD to FEF, the pathway mediating the attention effect is a matter of speculation. Assuming that the shift of attention was due to direct activation of the SC (and not, for example, due to antidromic activation of the FEF by way of its projections down to the $\mathrm{SC}$ ), the signals underlying the shift of attention must pass through the thalamus. One possibility is that the SC delay activity, which is thought to be related to directing visual attention, passes through MD, the same pathway that has been considered with respect to the corollary discharge. However, recall that in this SC-MD-FEF pathway the delay activity preceding a saccade decreased in MD compared to the same activity in the SC (Fig. 3B), making this pathway an unlikely conduit for delay activity. A more likely pathway is through the pulvinar. So although one particular pathway has been suspected as conveying this attentional signal, it is acknowledged that there is little physiological evidence for this so far.

This SC stimulation experiment thus provides another example of subcortical motor information modifying cortical activity. It is inferred that the SC stimulation affects visual processing in the cortex since the determination of direction of visual motion (the basis of the change blindness task) depends on identified areas of visual cortex, specifically extrastriate area MT (Newsome et al., 1985; Newsome and Pare, 1988). A recent report by Müller et al. (2005) appeared to confirm this.

\section{Conclusion: Motor drivers and sensory drivers}

Not only do higher order thalamic neurons receive collateral inputs from the output of the cerebral cortex, they also receive inputs from subcortical movement related areas as well. This study has considered whether these motor inputs are best regarded as modulatory inputs acting on the sensory based activity derived from the cortex or whether they 
are best regarded as driver inputs comparable in significance to the drivers studied so far in the sensory system. While much less is known about the ascending motor related activity than the activity ascending in the sensory pathways, the experimental evidence that has been considered in the SC to MD to FEF pathway provides some clues as to the nature of the input. The evidence from the visual-oculomotor activity seen in MD can be regarded as largely the result of driver input from SC. Another input from the SC to visual cortex has been shown to contribute to visual spatial attention, and this input via an as yet unidentified thalamic relay also is best viewed as a driver of thalamic activity rather than a modulator. Thus in Fig. 1 the dotted line representing ascending motor information from the midbrain should be a solid line indicative of a driver input. We conclude that the inputs to the higher order nuclei of the thalamus from the subcortical oculomotor areas should be regarded as motor drivers in parallel to the sensory drivers at the first order thalamic nuclei.

One consequence of this identification of a motor driver input to some higher order thalamic nuclei is the recognition of the potentially different information conveyed directly from one cortical region to another compared to the information conveyed indirectly from one cortical region to another through a higher order thalamic nucleus (Fig. 12). The higher order thalamic nucleus can convey information to a cortical area both from another cortical area via the descending pathway from layer 5 of cortex and from the ascending motor pathway. The pathway through the higher order nucleus offers the possibility of combining current information from cortex with new information on the impending movement. In contrast the direct cortical-cortical connections convey only information from the other cortical area. Thus these probably should not be regarded as alternate routes between successive cortical areas but rather as routes that contribute different information.

The drivers considered here are both essentially corollary discharges - they are copies of information in the motor pathway that are sent to the cerebral cortex - even though only the MD signal has been primarily referred to as a corollary discharge. There are other signals arriving at higher order thalamic nuclei, however, that could be driver inputs but not corollaries. For example, inputs to MD other than

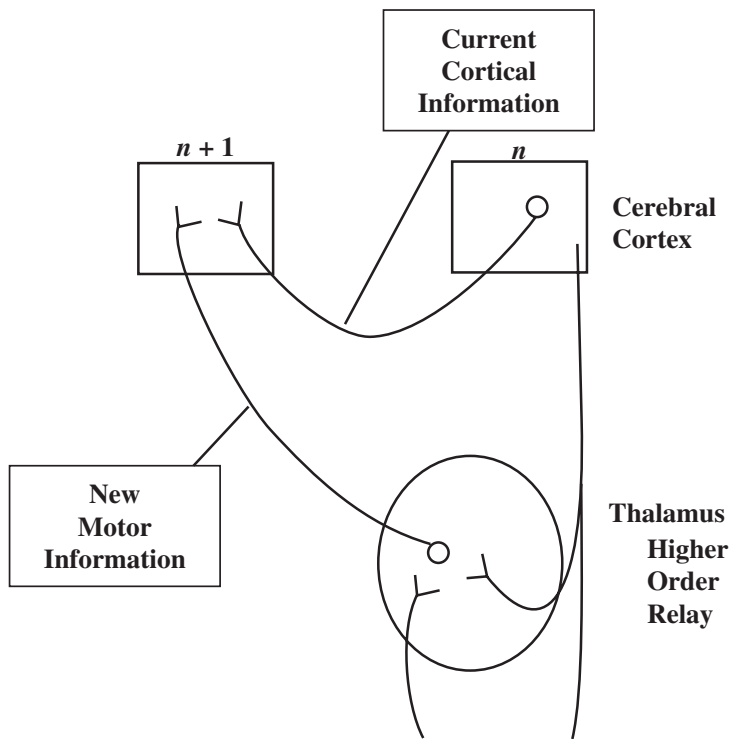

Fig. 12. Difference between the information conveyed to a cortical area from another cortical area and from a higher order thalamic nucleus. The higher order thalamic nucleus can convey both current information from another cortical area and new motor information from the ascending motor pathway. In contrast the direct cortical-cortical connections convey only the current information from the other cortical area.

those from the superior colliculus come from the cerebellum and the basal ganglia, and these may contribute to the control of movement rather than producing a corollary signal of that movement; thus far there is not enough evidence to decide (Sommer, 2003).

These motor drivers to the thalamus can be envisioned as the top of a series of loops in the primate brain that provide information to upper levels at the same time as they provide instructions to lower levels, usually, but not necessarily, for the control of movement (Fig. 13). Just as layer 5 of cortex can project into a subcortical area such as the SC and have a collateral to higher order thalamic nuclei, the SC can project to pontine areas and have a driving collateral that projects back to the thalamus. Note that at each step there is both a descending instruction and a collateral projection with a corollary of the instruction, with the exception that the thalamic nuclei project up to the cerebral cortex but do not themselves provide descending outputs. But this study considers the corollary conveyed by the 


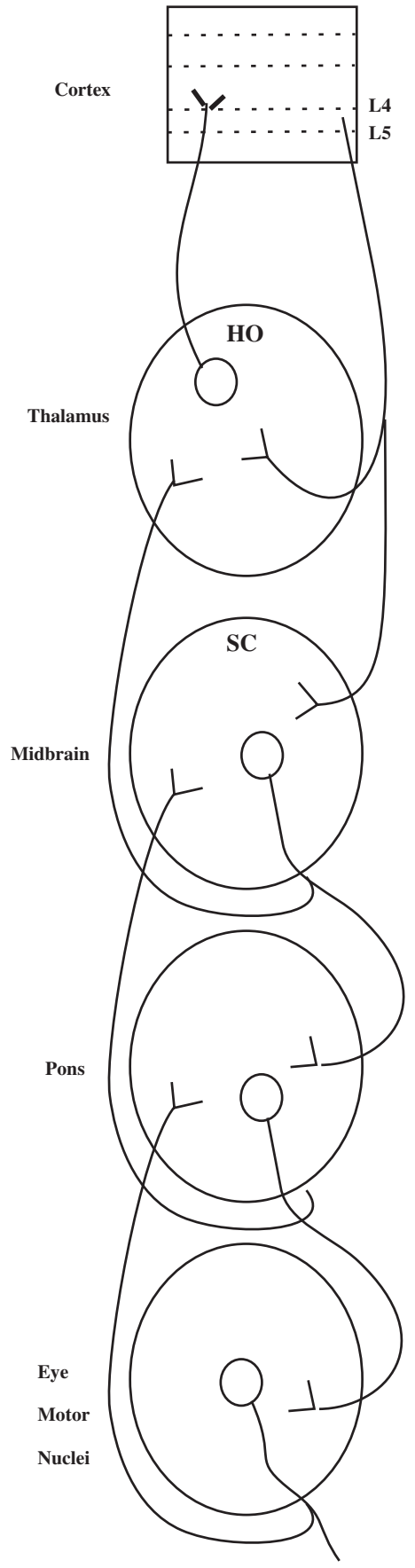

Fig. 13. Comparison of driver input and corollary discharge. The ascending collateral to the higher order nucleus of the thalamus from the SC conveys a corollary of the movement information sent downstream from the SC. It is suggested that this is one example of a pattern recurring repeatedly along the axis from cerebral processing to motor nuclei. thalamus as no different conceptually from the other corollaries below it on the path.

One ambiguous aspect of this discussion on drivers and modulators relates to the behavioral evidence that has been presented, specifically that the input from SC through thalamus modifies behavior once it reaches the cerebral cortex. If it ends up modifying behavior, in a rather subtle and covert way - e.g., through a shift of attention - why is the input at the cortex not considered a modulator? The hidden assumption in this question is that cortical processing is based primarily on sensory input, and that movementrelated input simply impinges on and modifies this processing. If this were true, the input received by the cortex from the SC via thalamus would appropriately be regarded as modulatory. But a great deal of evidence on the activity of neurons in the extrastriate visual areas, along with the parietal and frontal cortex, has shown that inputs other than the sensory make an equal if not larger contribution to neuronal activity. For example, in comparing activity in V1 and V4 in the extrastriate visual pathway, Haenny et al. (1988) found that neuronal activity became more determined by the monkey's set related to a search stimulus than to the stimulus itself. Therefore the assumption is made that cortical activity beyond the primary sensory areas is driven as much by processing and inputs that are distinct from the sensory input as by the sensory input itself. One of the major inputs is likely to be the input back to cortex from the higher order nuclei of the thalamus, as Guillery and Sherman have emphasized, and if the motor input to these thalamic nuclei is regarded as drivers, there is every reason to regard their contribution to cortical processing as much a driver as is the sensory input. In this sense the modification discussed here is a result of altering this mix of sensory and motor drivers, not just a modulation of the driver sensory input. Cortical processing should be regarded as resulting from inputs reporting the results of movement and/or prior processing as much as it results from current sensory input.

\section{References}

Basso, M.A. and Wurtz, R.H. (1998) Modulation of neuronal activity in superior colliculus by changes in target probability. J. Neurosci., 18: 7519-7534. 
Bell, A.H., Fecteau, J.H. and Munoz, D.P. (2004) Using auditory and visual stimuli to investigate the behavioral and neuronal consequences of reflexive covert orienting. J. Neurophysiol., 91: 2172-2184.

Benevento, L.A. and Fallon, J.H. (1975) The ascending projections of the superior colliculus in the rhesus monkey (Macaca mulatta). J. Comp. Neurol., 160: 339-361.

Cavanaugh, J. and Wurtz R. H. (2004) Subcortical modulation of attention counters change blindness. J. Neurosci., 24: $11236-11243$.

Dorris, M.C. and Munoz, D.P. (1995) A neural correlate for the gap effect on saccadic reaction times in monkey. J. Neurophysiol., 73: 2558-2562.

Fuster, J.M. (1997) The prefrontal cortex. Anatomy, Physiology, and Neuropsychology of the Frontal Lobe, 3rd edition, Lippincott-Raven, Philadelphia.

Ghose, G.M. and Maunsell, J.H.R. (2002) Attentional modulation in visual cortex depends on task timing. Nature, 419: 616-620.

Glimcher, P.W. and Sparks, D.L. (1992) Movement selection in advance of action in the superior colliculus. Nature, 355 : 542-545.

Goldman-Rakic, P.S. (1995) Cellular basis of working memory. Neuron, 14: 477-485.

Guillery, R.W. (1995) Anatomical evidence concerning the role of the thalamus in corticocortical communication: A brief review. J. Anat., 187(3): 583-592.

Guillery, R.W. (2003) Branching thalamic afferents link action and perception. J. Neurophysiol., 90: 539-548.

Guillery, R.W. and Sherman, S.M. (2002a) The thalamus as a monitor of motor outputs. Philos. Trans. R Soc. Lond. B Biol. Sci., 357: 1809-1821.

Guillery, R.W. and Sherman, S.M. (2002b) Thalamic relay functions and their role in corticocortical communication: Generalizations from the visual system. Neuron, 33: 163-175.

Haenny, P.E., Maunsell, J.H.R. and Schiller, P.H. (1988) State dependent activity in monkey visual cortex II. Retinal and extraretinal factors in V4. Exp. Brain Res., 69: 245-259.

Harting, J.K., Huerta, M.F., Frankfurter, A.J., Strominger, N.L. and Royce, G.J. (1980) Ascending pathways from the monkey superior colliculus: An autoradiographic analysis. J. Comp. Neurol., 192: 853-882.

Ignashchenkova, A., Dicke, P.W., Haarmeier, T. and Their, P. (2004) Neuron-specific contribution of the superior colliculus to overt and covert shifts of attention. Nat. Neurosci., 7: 56-64.

Jones, E.G. (2002) Thalamic organization and function after Cajal. Prog. Brain Res., 136: 333-357.

Kelly, L.R., Li, J., Carden, W.B. and Bickford, M.E. (2003) Ultrastructure and synaptic targets of thectothalamic terminals in the cat lateral posterior nucleus. J. Comp. Neurol., 464: 472-486.

Kuroda, M. and Price, J.L. (1991) Ultrastructure and synaptic organization of axon terminals from brainstem structures to the mediodorsal thalamic nucleus of the rat. J. Comp. Neurol., 313: 539-552.

Kustov, A.A. and Robinson, D.L. (1996) Shared neural control of attentional shifts and eye movements. Nature, 384: 74-77.

Lemon, R. (1984) Methods for neuronal recording in conscious animals. In: IBRO Handbook Series. Methods in the Neurosciences, Vol. 4. J. Wiley \& Sons, New York, pp. 95-102.

Lynch, J.C., Hoover, J.E. and Strick, P.L. (1994) Input to the primate frontal eye field from the substantia nigra, superior colliculus, and dentate nucleus demonstrated by transneuronal transport. Exp. Brain Res., 100: 181-186.

McAlonan, K., Brown, V.J. and Bowman, E.M. (2000) Thalamic reticular nucleus activation reflects attentional gating during classical conditioning. J. Neurosci., 20: 8897-8901.

McAlonan, K. and Wurtz, R.H. (2004) Cross modal attention modulation of thalamic reticular neurons in the macaque monkey. Soc. Neuroscience Abst. 175.8.

Montero, V.M. (1997) c-fos induction in sensory pathways of rats exploring a novel complex environment: Shifts of active thalamic reticular sectors by predominant sensory cues. Neuroscience, 76: 1069-1081.

Montero, V.M. (2000) Attentional activation of the visual thalamic reticular nucleus depends on "top-down" inputs from the primary visual cortex via corticogeniculate pathways. Brain Res., 864: 95-104.

Moore, T., Armstrong, K.M. and Fallah, M. (2003) Visuomotor origins of covert spatial attention. Neuron, 40: 671-683.

Müller, J.R., Philiastides, M.G. and Newsome, W.T. (2005) Microstimulation of the superior colliculus focuses attention without moving the eyes. Proc. Natl. Acad. Sci. USA, 102: $525-529$.

Newsome, W.T. and Pare, E.B. (1988) A selective impairment of motion perception following lesions of the middle temporal visual area (MT). J. Neurosci., 8: 2201-2211.

Newsome, W.T., Wurtz, R.H., Dursteler, M.R. and Mikami, A. (1985) Deficits in visual motion processing following ibotenic acid lesions of the middle temporal visual area of the macaque monkey. J. Neurosci., 5: 825-840.

Ramcharan, E.J., Gnadt, J.W. and Sherman, S.M. (2001) The effects of saccadic eye movements on the activity of geniculate relay neurons in the monkey. Vis. Neurosci., 18: 253-258.

Reichova, I. and Sherman, S.M. (2004) Somatosensory corticothalamic projections: Distinguishing drivers from modulators. J. Neurophysiol., 92: 2185-2197.

Reppas, J.B., Usrey, W.M. and Reid, R.C. (2002) Saccadic eye movements modulate visual responses in the lateral geniculate nucleus. Neuron, 35: 961-974.

Reynolds, J.H. and Desimone, R. (1999) The role of neural mechanisms of attention in solving the binding problem. Neuron, 24:19-29, 111-125. 
Rizzolatti, G. (1983) Mechanisms of selective attention in mammals. In: Ewert, J.-P., Capranica, R. and Ingle, D.J. (Eds.), Advances in Vertebrate Neuroethology. London, Plenum Publishing Corp.

Robinson, D.A. (1972) Eye movements evoked by collicular stimulation in the alert monkey. Vision Res., 12: 1795-1808.

Sheliga, B.M., Riggio, L. and Rizzolatti, G. (1994) Orienting of attention and eye movements. Exp. Brain Res., 98: 507-522.

Sherman, S.M. and Guillery, R.W. (2001) Exploring the Thalamus. Academic Press, San Diego, CA.

Sherman, S.M. and Guillery, R.W. (2002) The role of the thalamus in the flow of information to the cortex. Philos. Trans. R Soc. Lond. B Biol. Sci., 357: 1695-1708.
Sommer, M.A. (2003) The role of the thalamus in motor control. Curr. Opin. Neurobiol., 13: 663-670.

Sommer, M.A. and Wurtz, R.H. (2004a) What the brain stem tells the frontal cortex. I. Oculomotor signals sent from superior colliculus to frontal eye field via mediodorsal thalamus. J. Neurophysiol., 91: 1381-1402.

Sommer, M.A. and Wurtz, R.H. (2004b) What the Brain Stem Tells the Frontal Cortex. II. Role of the SC-MD-FEF Pathway in Corollary Discharge. J. Neurophysiol., 91: $1403-1423$.

Stepniewska, I., Qi, H.X. and Kaas, J.H. (1999) Do superior colliculus projection zones in the inferior pulvinar project to MT in primates? Eur. J. Neurosci., 11: 469-480.

Van Horn, S.C. and Sherman, S.M. (2004) Differences in projection patterns between large and small corticothalamic terminals. J. Comp. Neurol., 475: 406-415. 